\title{
Preparation of the $\mathrm{Pb}\left(\mathrm{Mg}_{1 / 3} \mathrm{Nb}_{2 / 3}\right) \mathrm{O}_{3}$ films by aqueous tape casting
}

\author{
Aylin Şakar-Deliormanlı ${ }^{\mathrm{a}, *}$, Erdal Çelik ${ }^{\mathrm{b}}$, Mehmet Polat ${ }^{\mathrm{a}}$ \\ ${ }^{a}$ Izmir Institute of Technology, Chemical Engineering Department, Urla, Izmir, Turkey \\ ${ }^{\mathrm{b}}$ Dokuz Eylul University, Metallurgy and Materials Engineering Department, Izmir, Turkey \\ Received 15 November 2007; received in revised form 24 May 2008; accepted 30 May 2008 \\ Available online 10 July 2008
}

\begin{abstract}
Lead magnesium niobate $(\mathrm{PMN})$ is a relaxor ferroelectric material. Because of its high dielectric constant and superior electrostrictive properties it is commonly used in the manufacture of multilayer electronic devices which is typically produced by tape casting. However, preparation of PMN slurry formulations to use in aqueous tape casting process is not investigated in detail yet. Therefore, in this study aqueous PMN formulations were developed for tape casting and its relation with the final properties of PMN films were investigated. The slurries were prepared using poly(acrylic acid)-based comb polymer as the dispersant, nonionic acrylic latex as the binder and the hydroxypropyl methyl cellulose as the wetting agent. The results showed that it is possible to prepare flexible, crack-free PMN films using highly concentrated suspensions without using any plasticizer. The study gives guidelines for the aqueous tape casting of PMN and can be adapted to processing of other multi-component metal oxides.
\end{abstract}

(C) 2008 Elsevier Ltd. All rights reserved.

Keywords: Tape casting; Suspensions; Perovskites; Niobates; Sintering

\section{Introduction}

Lead magnesium niobate (PMN) have important applications in multilayer electronic ceramic industry. ${ }^{1}$ Tape casting is a convenient method for manufacturing of thin and thick PMN sheets. With today's tape casting technology very thin tapes can be cast allowing manufacture of multilayer ceramic capacitors and transducers. ${ }^{2-3}$ In tape casting a paste-like suspension consisting of ceramic powder, solvent, dispersant, binder and plasticizer is cast onto a substrate by a moving blade at a constant distance to the substrate. The final dried green tape should be flexible, and easy to handle. ${ }^{4}$

Previously, organic solvents were preferred for the preparation of thin and thick films from ceramic suspensions due to their low surface tension. ${ }^{4,5}$ For example, Windlass et al. investigated the dispersion of lead magnesium niobate-lead titanate (PMN-PT) powders in slightly polar solvent, propylene glycol methyl ether acetate. ${ }^{6}$ Methyl ethyl ketone/ethanol, mesitylene, $\gamma$-butyrolactone, xylene, toluene, and isopropanol are other organic solvents has been used to prepare PMN-PT suspensions. ${ }^{7-9}$ However, it is

\footnotetext{
* Corresponding author. Tel.: +90 232 7507827; fax: +90 2327506210.

E-mail address: aylindeliormanli@iyte.edu.tr (A. Şakar-Deliormanlı).
}

necessary to move away from organic solvents towards waterbased systems due to health, environmental and economic considerations. ${ }^{10,11}$

The development of aqueous slurry formulations for the tape casting has been studied by many researchers for different ceramic materials. ${ }^{3,10,12-16}$ Doreau et al. investigated the processing of aqueous tape casting of alumina in the presence acrylic emulsion binders without using any additional organic additives in the system such as plasticizer or defoamer. ${ }^{12}$ Smay and Lewis developed an aqueous system for the tape casting of lead zirconate titanate. They investigated the effect of acrylic latex suspensions on this system in the presence of hydroxypropyl methylcellulose. ${ }^{13}$ Similarly, Ushifusa and Cima developed latex-based slurry for tape casting of mulliteglass composites suitable for electronic packaging. ${ }^{14}$ Thermal analysis and microstructural characterization of PMN films prepared by aqueous tape casting in the presence of acrylicbased binder was studied previously by Şakar-Deliormanlı et al. ${ }^{17}$

The aim of the current work is to prepare aqueous-based PMN slurries to use in tape casting process and investigate the final properties of the PMN films. The films were manufactured using a doctor blade having the gap height of $30-500 \mu \mathrm{m}$. The results of the study showed that it is possible to prepare high quality PMN films based on aqueous formulations. 


\section{Experimental}

\subsection{Materials}

Lead magnesium niobate, $\mathrm{Pb}\left(\mathrm{Mg}_{1 / 3} \mathrm{Nb}_{2 / 3}\right) \mathrm{O}_{3}$ powder which was produced by combustion spray pyrolysis method provided by Praxair Specialty Ceramics, Woodinville, WA, USA. Powder purity is $99.9 \%$ as reported by the manufacturer. Bulk density (Helium pycnometer Micromeritics 1330, Norcross, GA, USA), and the BET surface area (Model ASAP 2400, Micromeritics, Norcross, GA, USA) of the powder are measured to be $7.967 \mathrm{~g} / \mathrm{cm}^{3}$ and $1.168 \mathrm{~m}^{2} / \mathrm{g}$, respectively. Particle size distribution of the powder was obtained using a particle size analyzer (Model CAPA-700, Horiba, Ltd, Tokyo, Japan) and the $\mathrm{d}_{50}$ of the powder was measured to be $2.03 \mu \mathrm{m}$.

The poly(acrylic acid)-poly(ethylene)oxide PAA/PEO comb polymer (W.R. Grace and Co-Connecticut, Cambridge, MA) having a molecular weight of $25,800 \mathrm{~g} / \mathrm{mol}$ was employed as the dispersant to prepare stable PMN suspensions. A nonionic acrylic latex emulsion, Rhoplex B-60A (Rohm and Haas Co., Philadelphia, PA) having a glass transition temperature of $6{ }^{\circ} \mathrm{C}$ and a solids loading of $47 \mathrm{wt} . \%$ was employed as the binder. Hydroxypropyl methylcellulose (Methocel F4M, DOW Chemicals Co., Midland MI) with a molecular weight of $3500 \mathrm{~g} / \mathrm{mol}$ was used as the wetting agent. Methocel F4M contains $28 \%$ methoxyl with 1.8 degree of substitution and 5\% hydroxypropyl with 0.13 molar substitutions.

\subsection{Slurry preparation}

The tape casting slurries (total solids loading, 55 vol.\%) having different binder and hydroxypropyl methylcellulose (HPMC) concentrations were prepared by a multi-step process shown in Fig. 1. The slurry compositions used in tape casting are given in Table 1 . In the first step, highly concentrated PMN suspensions were prepared using PAA/PEO comb polymer. The optimum amount of dispersant necessary to disperse the PMN powders is $4.35 \mathrm{mg}$ polymer $/ \mathrm{m}^{2}$ of powder. ${ }^{18}$ The surface charge of PMN powders is positive (isoelectric point: 9.8 at 20 vol.\%) under basic conditions. Therefore, negatively charged PAA group of the comb polymer adsorb onto the PMN surface and creates electrosteric stabilization. ${ }^{19}$

Suspensions were ultrasonicated for $2.30 \mathrm{~min}$ (550 Sonic Dismembrator, Fisher Scientific) to break up the agglomerates in the system. Then they were ball milled for $24 \mathrm{~h}$ to ensure complete adsorption of PAA/PEO onto powder surface at $\mathrm{pH}$ 9. In the

Table 1

PMN slurry formulations used for the tape casting

\begin{tabular}{lcccc}
\hline & R1 (wt.\%) & R1.5 (wt.\%) & R2 (wt.\%) & R3 (wt.\%) \\
\hline PMN & 69.60 & 74.58 & 77.35 & 80.33 \\
Binder & 9.72 & 6.94 & 5.40 & 3.74 \\
Dispersant & 0.12 & 0.13 & 0.14 & 0.14 \\
Water & 20.46 & 18.25 & 17.03 & 15.71 \\
Wetting agent & 0.10 & 0.09 & 0.08 & 0.08 \\
Total & 100 & 100 & 100 & 100 \\
\hline
\end{tabular}

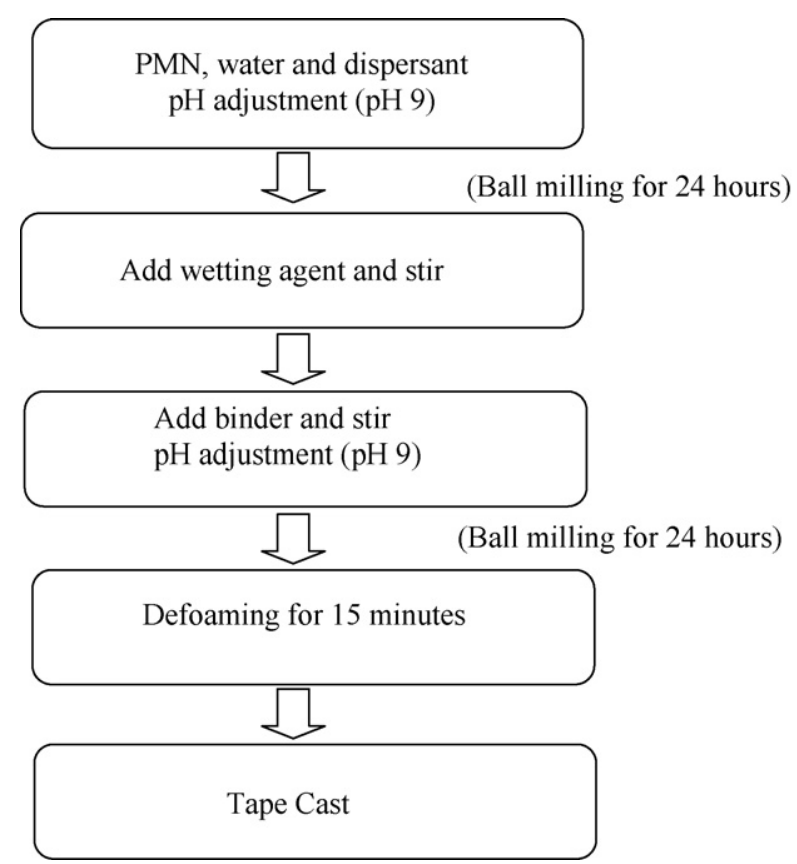

Fig. 1. Flow diagram showing the slurry preparation steps in tape casting process.

next step latex binder and wetting agent were added to the system and the slurry was ball milled for a further $24 \mathrm{~h}$. The $\mathrm{pH}$ was readjusted to 9.0 using $1 \mathrm{M} \mathrm{NH}_{4} \mathrm{OH}$. The slurry was defoamed at $2000 \mathrm{rpm}$ for 15 min using a planetary centrifugal mixer (Thinky ARE-250) prior to casting.

\subsection{PMN film fabrication}

PMN films were produced using laboratory type tape caster (Cladan Inc. Model 133, Serial 181, USA). The slurry was cast onto a silicone coated poly(ethylene terephthalate) stationary film (Mylar, PET blend EI-8526, Douglas-Hanson, Hammond, WI). Silicon coating having thickness of $1.27 \mu \mathrm{m}$ is on one side of the film. Surface energy of the film is $23 \mathrm{~mJ} / \mathrm{m}^{2}$ as reported by the Smay and Lewis. ${ }^{13}$ Through the experiments the casting rate was $25 \mathrm{~mm} / \mathrm{s}$ over a length of $1 \mathrm{~m}$. Doctor blade head having width of $100 \mathrm{~mm}$ was set at a height from $30 \mu \mathrm{m}$ to $500 \mu \mathrm{m}$. After deposition the tapes were allowed to dry in air at $25^{\circ} \mathrm{C}$ for $24 \mathrm{~h}$.

\subsection{Lamination and sintering}

Arrays of samples were cut along the length of dried tape. Samples were formed by laminating the 15 layers of green tapes between parallel plates. Then they were heated to $80^{\circ} \mathrm{C}$ and kept at this temperature for $1 \mathrm{~h}$. After heat treatment $30 \mathrm{MPa}$ pressure was applied to the samples for $2 \mathrm{~min}$.

To investigate the optimum sintering conditions some preliminary experiments were performed using PMN pellets. For this purpose, PMN powders containing $3 \mathrm{wt}$.\% polyvinyl alcohol were die pressed and then sintered at various temperatures ranging from $1000^{\circ} \mathrm{C}$ to $1275^{\circ} \mathrm{C}$. For the sintering of PMN films following procedure was applied: The binder was burned out by heating the samples at $1{ }^{\circ} \mathrm{C} / \mathrm{min}$ to $350^{\circ} \mathrm{C}, 1 \mathrm{~h}$ dwell 
at $350^{\circ} \mathrm{C}$ and $3{ }^{\circ} \mathrm{C} / \mathrm{min}$ to various temperatures ranging from $1000^{\circ} \mathrm{C}$ to $1250^{\circ} \mathrm{C}, 2 \mathrm{~h}$ dwell at the specified temperature. During heat treatment, alumina crucible system with cover was used in order to prevent the lead loss from the samples. To provide a lead rich atmosphere lead zirconate $\left(\mathrm{PbZrO}_{3}\right)$ powder bed was used inside the crucible.

\subsection{Characterizations}

Rheological measurements were performed using a stresscontrolled rheometer (Bohlin CS-50, Sweden) with C14 geometry (cup inner diameter: $16 \mathrm{~mm}$ and bob outer diameter: $14 \mathrm{~mm}$ ) at a constant temperature of $25^{\circ} \mathrm{C}$. A special designed solvent trap was used to minimize the evaporation during the measurements. The apparent viscosity of the samples was measured as a function of shear stress in ascending order.

Contact angle measurements were performed using a contact angle measuring instrument (Kruss DSA10 Contact Angle Measuring System). Contact angle of the samples on the Mylar film (silicon coated side) were measured using the sessile drop method. For each sample five different measurements were performed and average of these values was reported. Surface Tension measurements were performed by a tensiometer (KrussDigital K 10ST) using Du Nouy ring method. Platinum ring geometry was heated in a flame until it glowed red before each measurement to eliminate a possible contamination.

The roughness of the green films was analyzed using a surface profilometer (Mitutoya Surftest SJ-201 P/M). Through the measurements, profilometer sampling length and cut off length was adjusted as $5 \mathrm{~mm}$ and $0.8 \mathrm{~mm}$, respectively. Measurements were performed on PMN films with $5 \mathrm{~cm}$ arrays through the entire film length.

Microstructure of the green and sintered tapes were characterized by scanning electron microscope SEM (Philips, XL-30S FEG). Crystal structures were analyzed using X-ray diffractometer (Philips, X'pert Pro).

Dielectric constant of PMN samples were measured using a high resolution dielectric analyzer (Novo Control, Alpha-N). For the measurements a silver (Ag) paste was applied to the both sides of the sintered pellets and PMN films to improve the contact with the analyzer plates. A heat treatment at $600{ }^{\circ} \mathrm{C}$ was applied to the silver paste coated samples for $30 \mathrm{~min}$. Relative permittivity of the PMN samples were measured at room temperature as a function of frequency in the range $10^{-2} \mathrm{~Hz}$ to $10^{5} \mathrm{~Hz}$. During the measurements AC volts ( $3 \mathrm{Vrms}$ ) was applied to the samples.

\section{Results and discussion}

\subsection{Solution properties}

The apparent viscosity as a function of shear stress of pure latex suspensions of varying solids loading are shown in Fig. 2a. As it is clearly indicated in the graph emulsions exhibited Newtonian behavior at low solids content $\phi<0.3$. However, above $\phi: 0.3$ a transition to shear thinning was observed and the degree of shear thinning increased with increasing solids concentration $(\phi)$. According to Fig. 2a shear thinning behavior was dominant at $47 \mathrm{wt} . \%$. Such a behavior is in good agreement with the requirements for the tape casting process. Because the relatively high shear stress enables the slurry to have a low viscosity under the blade during casting, facilitating the tape casting. After deposition, the slurry recovers a high viscosity level, avoiding the settling of particles along the tape thickness. ${ }^{12}$

The zeta potential values of nonionic acrylic emulsion of B-60A is given in Fig. $2 \mathrm{~b}$ as a function of $\mathrm{pH}$. Although it is a nonionic emulsion, results showed that isoelectric point (IEP) of $\mathrm{B}-60 \mathrm{~A}$ is lower than $\mathrm{pH} 2$. Based on the previous study of Brown and Garrett, B-60A is an emulsion containing acrylic copolymer and nonionic emulsifier. ${ }^{20}$ Nahass et al. ${ }^{21}$ reported that Rhoplex B-60A is likely a copolymer between methyl methacylate and another acrylic monomer such as ethyl acrylate. ${ }^{21}$ Similarly, Ushifusa and Cima determined the isoelectric point of B-60A at about $\mathrm{pH} 2$ in absence of any electrolyte. ${ }^{14}$

Fig. 3 shows the viscosity of the hydroxypropyl methylcellulose solution as a function of concentration. It is clear that starting from $5 \mathrm{mg} / \mathrm{ml}$ the viscosity of the solution increases dramatically. This increase may also affect the viscosity of the PMN slurry to be used in tape casting. The results showed that HPMC can be used also as a thickening agent to modify the viscosity of the PMN suspensions.
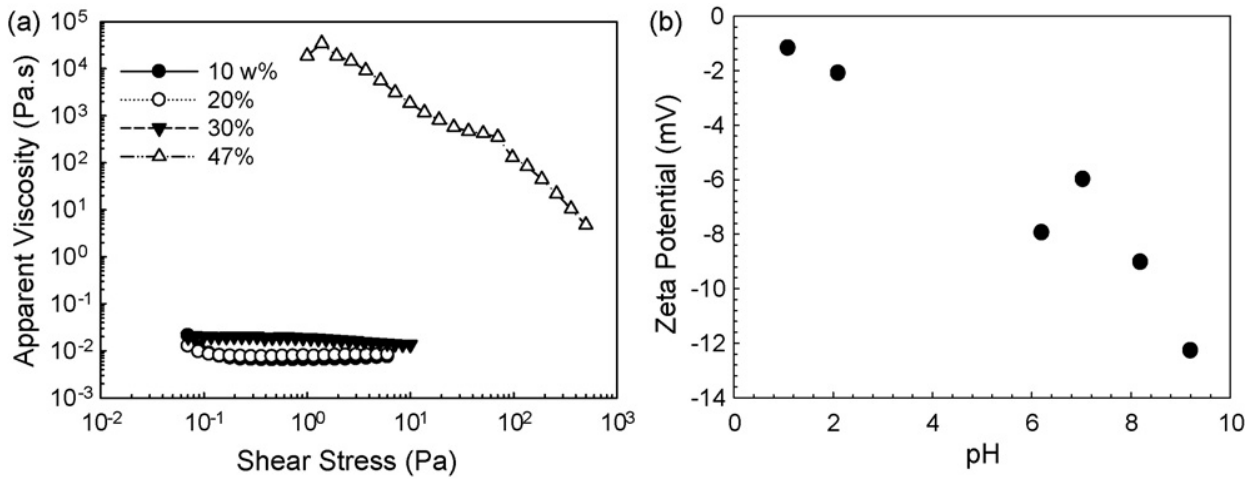

Fig. 2. (a) Log-log plot of apparent viscosity as a function of shear stress for pure latex suspension (B-60A) of varying concentrations. (b) Zeta potential vs. pH of binder B-60A solution (10 vol.\%). 


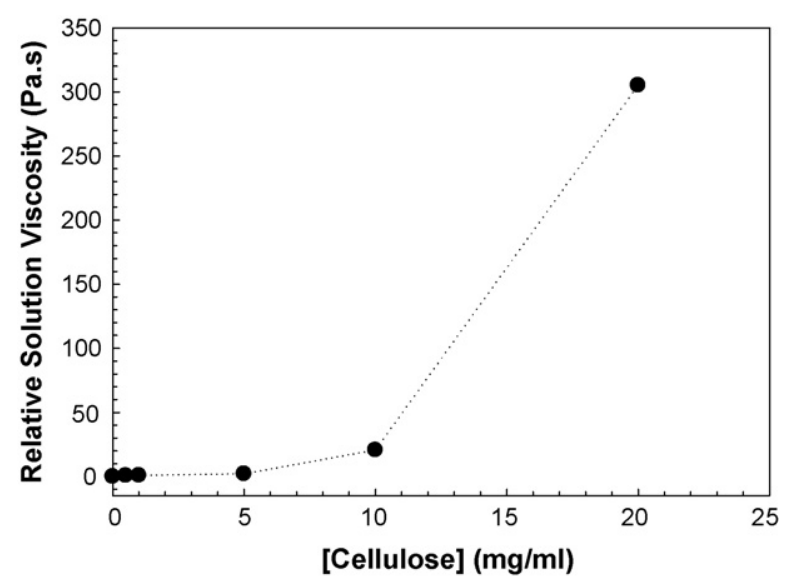

Fig. 3. Relative viscosity of HPMC solution as a function of concentration.

\subsection{Rheology of PMN slurries}

Rheological behavior of PMN slurries was investigated as a function of binder and the wetting agent (HPMC) content. Fig. 4 shows the effect of composition on the apparent viscosity of the tape casting suspensions ( $\left.\phi_{\text {tot }}: 0.55\right)$. According to the figure there is a gradual increase in viscosity as the binder concentration increase. It is also crucial to investigate the optimum cellulose concentration in PMN slurries both for deposition and spreading issues. Because, at very high cellulose concentrations decrease in viscosity may be observed due to variation in solids loading. The effect of cellulose additions on suspension rheology is illustrated in Fig. 5. The figure indicates that the viscosity of the PMN slurries increase as the cellulose concentration increases. The viscosity reaches to $2 \times 10^{4} \mathrm{~Pa}$ s at low shear region in the presence of $10 \mathrm{mg} / \mathrm{ml} \mathrm{HPMC}$. However, it is important to note that during tape casting process generally a higher shear stress is applied to the samples. Therefore, viscosity values in high shear region should be considered (see inset in Fig. 5).

In the study the viscoelastic behavior of slurries were examined by dynamic tests. Such experiments must be carried out at small amplitude of oscillation, so that the test conditions can be considered as nondestructive. In the case of concentrated sus-

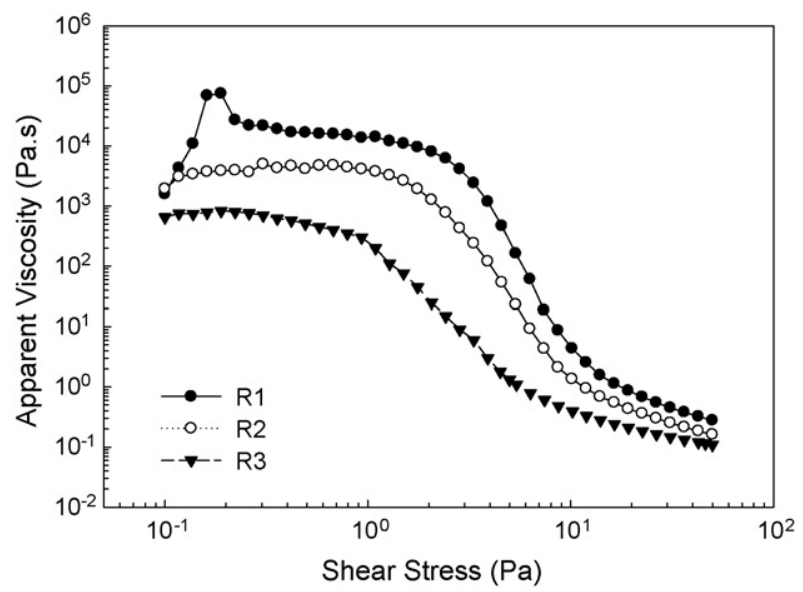

Fig. 4. Viscosity of slurries having different PMN/binder ratio as a function of shear stress, $\phi_{\text {tot }}: 0.55$.

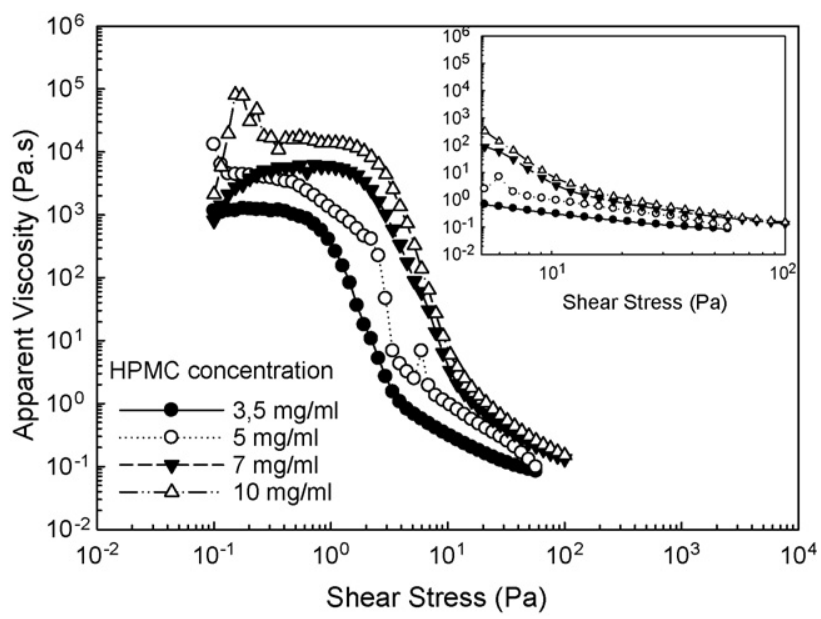

Fig. 5. Effect of HPMC concentration on the viscosity of slurry R1 as a function of shear stress, $\phi_{\mathrm{tot}}: 0.55$.

pensions like tape casting slurries the linear viscoelastic region is often limited in the very low strain amplitudes. ${ }^{22}$ Fig. 6 shows the elastic $\left(G^{\prime}\right)$ and loss or viscous modulus $\left(G^{\prime \prime}\right)$ of the PMN slurry depending on the shear stress amplitude. The elastic modulus of the slurry reaches a maximum value of $180 \mathrm{~Pa}$ in low shear region. This is caused by the high solids content which increases the elastic properties of the slurry. At low shear stresses the modulus remained nearly constant and $G^{\prime}$ was larger than $G^{\prime \prime}$, which means that the slurry behaves more elastic than viscous. The storage module $G^{\prime}$ exhibited a sudden decrease at a critical shear stress, $\tau_{\mathrm{c}}$. Because above $\tau_{\mathrm{c}}$ weak attractive forces between the powder and/or binder particles are broken up by the external shear stress and this destroys the internal network. The elastic properties of the slurry become very low and the storage modulus decreases above this critical stress. The loss modulus $G^{\prime \prime}$ reaches a maximum at this point and the energy which is used to break the networks cannot be stored as elastic deformation. ${ }^{23,24}$ The slurry must spread the carrier film during tape casting process. This means a shear stress greater than $\tau_{\mathrm{c}}$ must be applied to the slurry. In other word, for tape casting the critical shear stress should be high enough which could not result in sedimen-

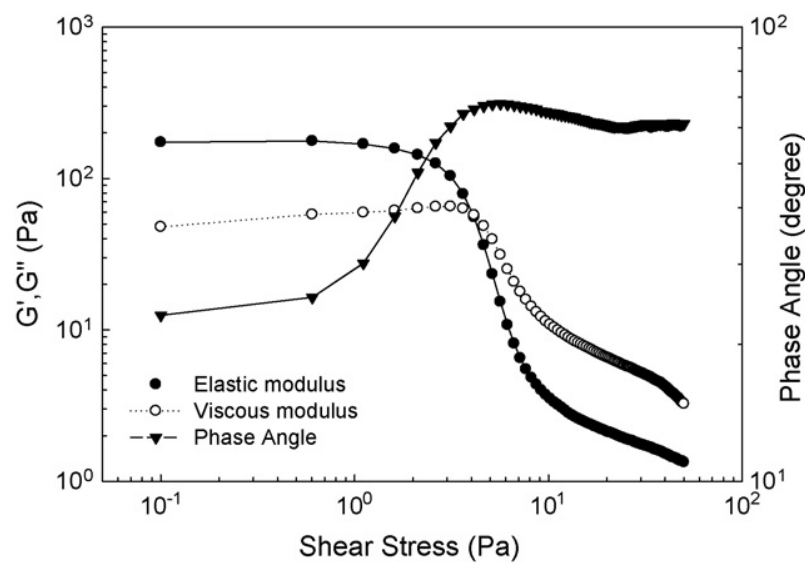

Fig. 6. Viscoelastic properties of slurry R1 as a function of shear stress, HPMC $(7 \mathrm{mg} / \mathrm{ml})$ and $\phi_{\text {tot }}: 0.55$. 


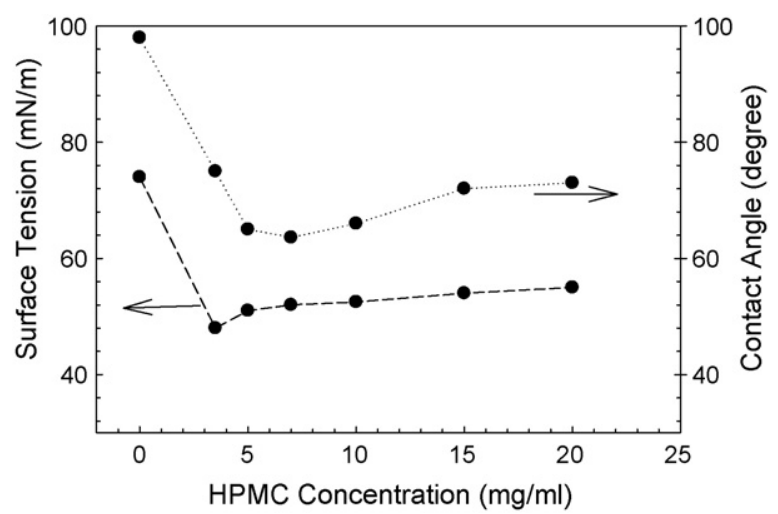

Fig. 7. Surface tension of HPMC solution and contact angle of with Mylar film as a function of concentration.

tation of the particles. On the other hand, $\tau_{\mathrm{c}}$ must not be too high because the slurry must flow under the shear conditions which occur during passing the doctor blades. ${ }^{25}$

To investigate the thixotropic behavior of the PMN slurries the viscosity was measured as a function of shear rate in the range of $0.1-700 \mathrm{~s}^{-1}$ in an up and down sweep. A good superposition of up and down curves was obtained in the whole shear rate range. Similarly, time-dependent behavior of the PMN slurries was also examined by oscillation measurements. During the time of oscillation $\left(10^{3} \mathrm{~s}\right)$ almost no changes in internal structure of the slurries were observed. The storage and loss modulus remained nearly constant during this period.

\subsection{Wetting behavior}

In the study, a silicone-coated Mylar film was used for slurry deposition. The main purpose of the silicone coating was to facilitate the easy release of the dried tape. However, suspensions experienced strong de-wetting forces during casting onto these films. De-wetting phenomena is influenced by both surface energy and viscosity. ${ }^{13}$ The other parameter that influence wetting is the contact angle between solid and liquid. ${ }^{13,26}$ Therefore, to improve shape retention of as-cast layers, the effects of reducing the surface tension and enhancing the low shear viscosity of this system should be investigated. In Fig. 7 surface tension of cellulose solution and contact angle between cellulose

Table 2

Contact angle values of water, binder and PMN suspensions (slurry composition R1) with the Mylar film

\begin{tabular}{ll}
\hline Sample & Contact angle $\left(^{\circ}\right)$ \\
\hline Water-glass & 19 \\
Water-Mylar film & 94 \\
Binder (B60A)-Mylar film & 63 \\
R1(3.5) & 64 \\
R1(5) & 64 \\
R1(6) & 54 \\
R1(7) & 52.5 \\
R1(8) & 52.5 \\
R1(10) & 66
\end{tabular}

Values in parenthesis refer to HPMC concentration in $\mathrm{mg} / \mathrm{ml}$. and carrier film are shown as a function of cellulose concentration. According to Fig. 7, surface tension and contact angle of cellulose much lower compared to the corresponding values of water. With increasing polymer concentration thereby increasing viscosity, slight increased was observed in the measured contact angle values. Consequently, spreading of the solutions on the Mylar film surface decreased. Riedl et al. investigated the surface tension and contact angle of HPMC in the absence and presence of plasticizer. Surface tension value of HPMC (2 wt.\%) was reported to be $52.88( \pm 1.4) \mathrm{mN} / \mathrm{m} .{ }^{27}$ Therefore, there is an agreement between the surface tension values obtained in this study and the literature values. Contact angle between the carrier film and the PMN slurries having R1 composition (fixed pow$\mathrm{der} /$ binder ratio) with varying cellulose concentrations $(\mathrm{mg} / \mathrm{ml})$ are given in Table 2. Accordingly, at cellulose concentration between 7 and $8 \mathrm{mg} / \mathrm{ml}(0.1 \mathrm{wt} . \%)$ the contact angle between the PMN slurry and the carrier film is lower compared to the values at other concentrations.

\subsection{Drying behavior and roughness of PMN films}

During drying stresses may develop in ceramic films. As the water evaporates the film shrinks in the $z$-axis however shrinkage in other axes is limited due to adhesion to the substrate. This may cause stress formation on the film. ${ }^{28,29}$ The results of this study showed that some cracks formed on the film having a composition of R2 during drying period. At higher binder content (R1.5), perpendicular cracks were observed on the surface of the film with respect to casting direction. On the other hand, in the films prepared from R1 composition ( $7 \mathrm{mg} / \mathrm{ml}$ HPMC) crack formation was not observed during drying period. PMN films having dried green thickness of 10-265 $\mu \mathrm{m}$ were produced successfully using this composition. The stress development and crack formation during drying of PMN films was attributed to the moisture gradient in green bodies and low binder concentration in slurry composition. Because stresses cause formation of cracks unless relatively large amounts of organic binders added. Differential shrinkage between the top and bottom of the film produce stress that results in cracks. Additionally, par-

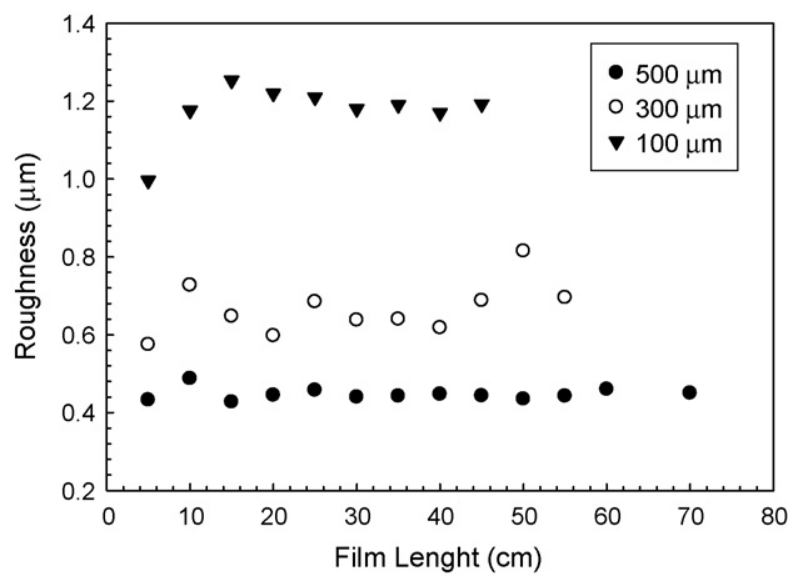

Fig. 8. Roughness of the PMN films prepared from slurry composition of R1 as a function of film thickness (in wet state). 


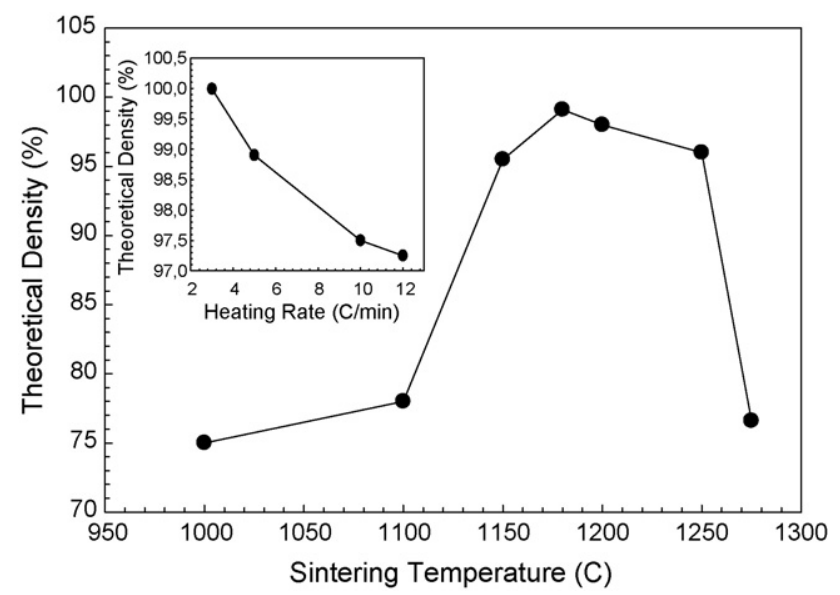

Fig. 9. Sintered density of the PMN pellets compared to the theoretical one as a function of sintering temperature. Inset plot: Effect of heating rate on the sintering density of PMN pellets, sintering at $1180^{\circ} \mathrm{C}$ for $2 \mathrm{~h}$.

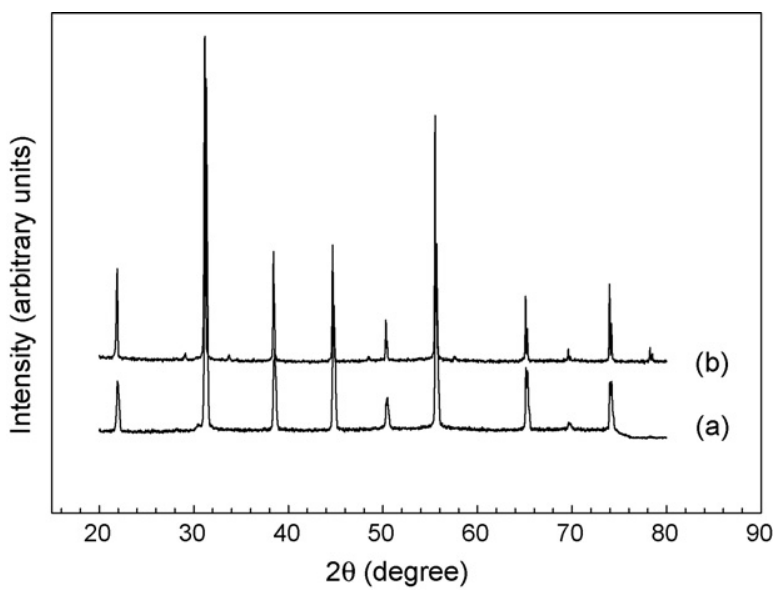

Fig. 10. X-ray diffraction patterns of the PMN films sintered at: (a) $1180{ }^{\circ} \mathrm{C}$ and (b) $1250^{\circ} \mathrm{C}$.

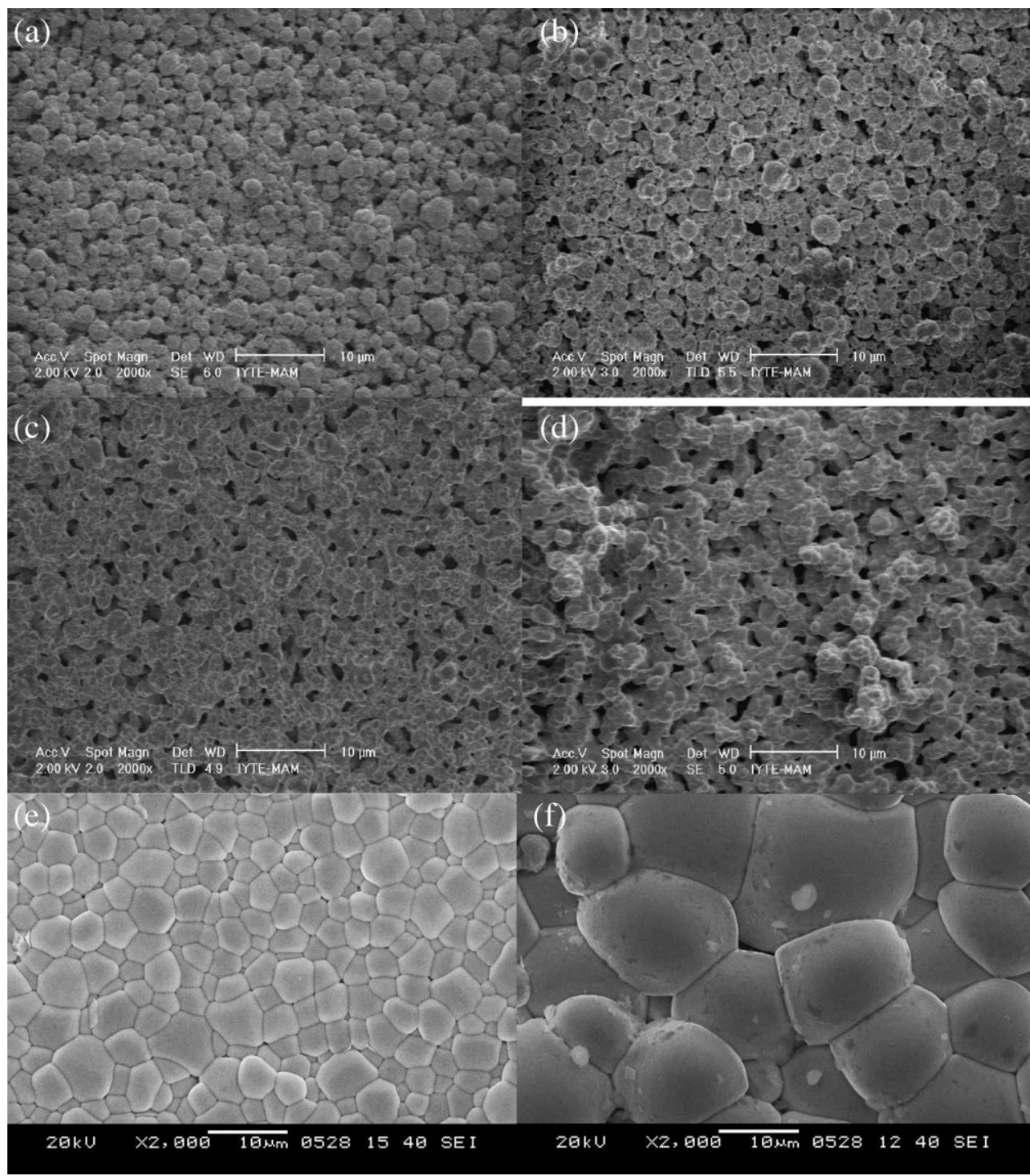

Fig. 11. SEM micrographs of the PMN films sintered at different temperatures: (a) $575^{\circ} \mathrm{C}$; (b) $1000^{\circ} \mathrm{C}$; (c) $1100^{\circ} \mathrm{C}$; (d) $1150^{\circ} \mathrm{C}$; (e) $1180^{\circ} \mathrm{C}$; (f) $1250^{\circ} \mathrm{C}$. 
ticle rearrangement may bring some stress in the thickness direction. ${ }^{13,29-31}$

Surface profilometry analysis was performed in order to investigate the roughness of the dried PMN films through the entire film length. Fig. 8 depicts the effect of film thickness on the roughness of the PMN green sheets. The results showed that roughness of the films prepared from slurry composition of R1 ( $7 \mathrm{mg} / \mathrm{ml} \mathrm{HPMC)}$ is dependent on the film thickness. As the thickness of the film decreased an increase was observed on the roughness of these films.

\subsection{Sintering and microstructural evolution}

High density products are generally desired in ceramic processing and it is a requirement in the production of electronic ceramic components. ${ }^{32}$ Therefore, sintering of the green tapes has a significant importance in tape casting process. Fig. 9 shows the sintered density (compared to the theoretical density) of PMN pellets as a function of sintering temperature. Densities were determined by measuring the weight and dimensions of the sintered samples. The measured density of the PMN pellets after sintering at $1180{ }^{\circ} \mathrm{C}$ for $2 \mathrm{~h}$ was about $7.865 \mathrm{~g} / \mathrm{cm}^{3}$ representing the highest value $(99 \%)$ compared to the theoretical density. This sintering temperature was thus chosen also for the tape samples. A sharp decrease was observed in density values of the pellets sintered at $1275^{\circ} \mathrm{C}$. The reason of this decline was attributed to the significant lead evaporation from the samples that cause formation of pyrochlore phase. The results also revealed that the sintered density of the PMN films prepared from a slurry composition of R1 was $7.568 \mathrm{~g} / \mathrm{cm}^{3}$ (95\% of the theoretical density) after a heat treatment at $1180^{\circ} \mathrm{C}$ for $2 \mathrm{~h}$. Dried green densities of these films were $58-60 \%$ of the theoretical density. Inset in Fig. 9 presents the effect of heating rate on the density of PMN pellets sintered at $1180{ }^{\circ} \mathrm{C}$ for $2 \mathrm{~h}$. The results showed that highest density was obtained at a heating rate of $3{ }^{\circ} \mathrm{C} / \mathrm{min}$ and at higher heating rates a gradual decrease was observed in densities.

Fig. 10 demonstrates the effect of sintering temperature on the crystal structure of PMN films. According to the XRD diagram, the film under investigation has a pure perovskite structure after sintering at $1180^{\circ} \mathrm{C}$ for $2 \mathrm{~h}$. On the other hand, few low intensity pyrochlore peaks were detected in the sample that is sintered at $1250{ }^{\circ} \mathrm{C}$ for $2 \mathrm{~h}$.

Microstructures of the PMN films sintered at different temperatures are given in Fig. 11. According to Fig. 11a-d, PMN films sintered at $575-1150{ }^{\circ} \mathrm{C}$ have porous structures. On the other hand, Fig. 11e shows that it is possible to obtain high-density PMN films having homogeneous microstructure at $1180^{\circ} \mathrm{C}$. At higher sintering temperatures abnormal grain growth and the formation of a second phase initiate. The crosssectional view of the PMN films sintered at $1180^{\circ} \mathrm{C}$ for $2 \mathrm{~h}$ is given in Fig. 12a-d. Accordingly, PMN films cast with a doctor blade at a gap height of $30 \mu \mathrm{m}$ have a final thickness of $5 \mu \mathrm{m}$ after sintering at $1180^{\circ} \mathrm{C}$ for $2 \mathrm{~h}$ (Fig. 12a).

\subsection{Electrical properties}

In the study relative permittivity of the PMN pellets and films were measured as a function of frequency. It is known that, dielectric constant of PMN is maximum at about $-10^{\circ} \mathrm{C}$
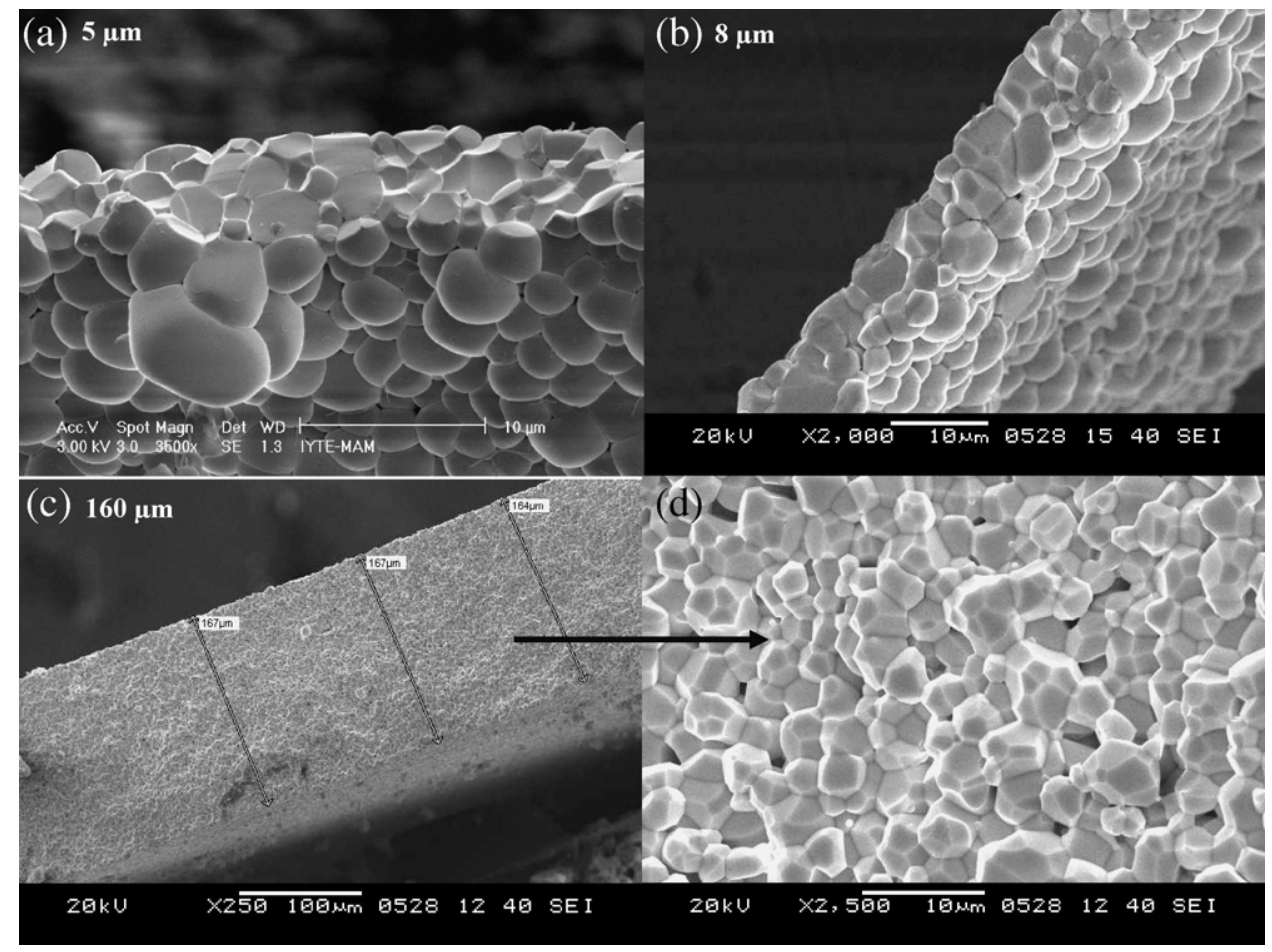

Fig. 12. SEM micrographs showing the final thickness and the microstructure of the PMN films sintered at $1180^{\circ} \mathrm{C}$ for $2 \mathrm{~h}$. (a) Final thickness, $5 \mu \mathrm{m}$; (b) final thickness, $8 \mu \mathrm{m}$; (c) final thickness, $160 \mu \mathrm{m}$; (d) cross-section of the film $(160 \mu \mathrm{m})$ at higher magnification. 


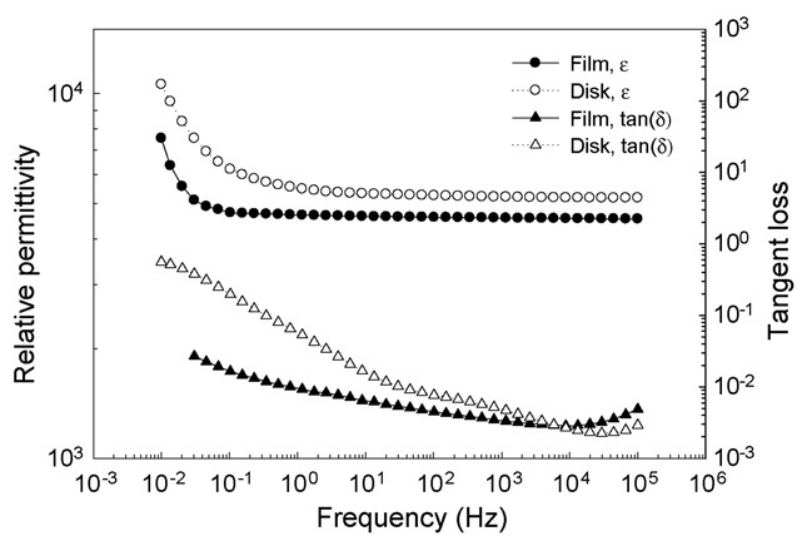

Fig. 13. Relative permittivity and the tangent loss of the PMN pellets and films as a function of frequency.

and above this temperature dielectric constant decreases with increasing temperature. ${ }^{33}$ In this work, measurements were performed at room temperature $\left(25 \pm 2^{\circ} \mathrm{C}\right)$ therefore results do not corresponds to the maximum relative permittivity values for these samples. Accordingly, Fig. 13 describes the relative permittivity, $\varepsilon_{\mathrm{r}}$, and the tangent loss of the PMN samples as a function of frequency. It is obvious that $\varepsilon_{\mathrm{r}}$ of the PMN samples is dependent on the frequency. Relative permittivity of the PMN pellets were measured to be 11,000 at $0.01 \mathrm{~Hz}$. Similarly, relative permittivity of the PMN films sintered at $1180^{\circ} \mathrm{C}$ is 8000 at $0.01 \mathrm{~Hz}$. Results showed that under the same conditions $\varepsilon_{\mathrm{r}}$ values of the PMN bulk samples are higher compared to the films. Previous studies related to the electrical characterization of ferroelectric thick films showed that films may have a lower relative permittivity compared to the bulk ceramics. ${ }^{33}$ For example, Gentil et al. measured the relative permittivity of PMN films as 10,000 at $0.1 \mathrm{kHz}$ which is lower than the values of the bulk ceramics $(17,800$ at $0.1 \mathrm{kHz})$ under the same conditions. They also found that the decrease of film thickness lead to the decrease of dielectric properties. ${ }^{33}$ In this study the lower relative permittivity values in PMN thick films compared to the bulk samples may be attributed to the several factors, including higher porosity, higher roughness, presence of pyrochlore phase or some void formation between the layers.

\section{Conclusions}

PMN slurry formulations were developed to use in aqueous tape casting process. Since PMN is partially soluble in water ion dissolution during wet processing strongly affects the colloidal properties of suspensions. For the slurry preparation PAA/PEO comb polymer was employed as the dispersant since it is effective in systems containing dissolved multivalent metal ions. Effect of binder and the wetting agent concentration on the wetting/spreading behavior of PMN slurries and the drying properties of PMN films were investigated. The results revealed that films prepared from a suspension composition of R1 did not experience any problem during casting, drying and sintering steps. After sintering at $1180^{\circ} \mathrm{C}$ films having thickness down to $5 \mu \mathrm{m}$ were obtained. The results showed that it is possible to prepare flexible, crack free PMN films having high relative permittivity values after sintering using highly concentrated aqueous suspensions.

\section{Acknowledgements}

The authors would like to thank Prof. Dr. Jennifer A. Lewis for her support and use of the laboratory facilities at University of Illinois at Urbana Champaign, USA. Contributions of Dr. James Smay are gratefully acknowledged. The project is supported by the State Planning Organization of Turkey (DPT) and The Scientific and Technological Research Council of Turkey (TUBITAK, NATO-A2).

\section{References}

1. Tzou, H. S., Lee, H. J. and Arnold, S. M., Smart materials, precision sensors/actuators, smart structures, and structronic systems. Mech. Adv. Mater. Struct., 2004, 11, 367-393.

2. Safari, A., Allahverdi, M. and Akdogan, E. K., Solid freeform fabrication of piezoelectric sensors and actuators. J. Mater. Sci., 2006, 41, 177-198.

3. Tian, T., Jiang, D., Zhang, J. and Lin, Q., Aqueous tape casting process for hydroxyapatite. J. Eur. Ceram. Soc., 2007, 27, 2671-2677.

4. Meier, P. L., Urech, L. and Gauckler, L. J., Tape casting of nanocrystallize ceria gadolinia powder. J. Eur. Ceram. Soc., 2004, 24, 3753-3758.

5. Jantunen, H., Hu, T., Uusimaki, A. and Vuori, S. L., Tape casting of ferroelectric, dielectric piezoelectric and ferromagnetic materials. J. Eur. Ceram. Soc., 2004, 24, 1077-1081.

6. Windlass, H., Raj, P. M., Balaraman, D., Bhattacharya, S. K. and Tummala, R. R., Polymer-ceramic nanocomposite capacitors for system-on-package (SOP) applications. IEEE Trans. Adv. Pack., 2003, 26, 10-16.

7. Agarval, V., Chahal, P., Tummala, R. R. and Allen, G. M., Improvements and recent advances in nanocomposite capacitors using a colloidal technique. In IEEE, Proceedings of the Electronic Components and Technology Conference, 1998, pp. 165-170.

8. Ogitani, S., Bidstrup-Allen, S. and Kohl, P., Development of high-K photodefinable epoxy composites for embedded capacitors. In IEEE, ICPMT Proceedings of International Electronics Manufacturing Technology Symposium, 1998, pp. 199-205.

9. Vasanthakumari, K. G., Denny, A. P., Sasidharan, K., Unnikrishnan, G., Raghu, N. and Pizoelectric, Electrostrictive multilayer actuators. In Proceedings of ISSN, International Conference on Smart Structures and Systems, 2005, pp. 129-136.

10. Feng, J.-H. and Dogan, F., Aqueous processing and mechanical properties of PLZT green tapes. Mater. Sci. Eng., 2000, A283, 56-64.

11. Mistler, R. E., Tape casting: past, present potential. Am. Ceram. Soc. Bull., 1998, 77, 82-86.

12. Doreau, F., Tari, G., Pagnoux, C., Chartier, T. and Ferreira, J. M. F., Processing of aqueous tape cating of alumina with acrylic emulsion binders. $J$. Eur. Ceram. Soc., 1998, 18, 311-318.

13. Smay, J. E. and Lewis, J. A., Structural and property evolution of aqueous based lead zirconate titanate tape cast layers. J. Am. Ceram. Soc., 2001, 84, 2495-2500.

14. Ushifusa, N. and Cima, M. J., Aqueous processing of mullite-containing green sheets. J. Am. Ceram. Soc., 1991, 74, 2443-2447.

15. Pagnoux, C., Chartier, T., Granja, F., Doreau, F., Ferreira, J. M. and Baumard, J. F., Aqueous suspensions for tape casting based on acrylic binders. J. Eur. Ceram. Soc., 1998, 18, 241-247.

16. Kristofferson, A., Roncari, E. and Galassi, C., Comparison of different binders for water based tape casting of alumina. J. Eur. Ceram. Soc., 1998, 18, 2123-2131.

17. Şakar-Deliormanlı, A., Çelik, E. and Polat, M., Thermal analysis and microstructural characterization of ceramic green tapes prepared by aqueous tape casting. J. Therm. Anal. Calorim., in press. 
18. Şakar-Deliormanlı, A., Çelik, E. and Polat, M., Viscosity and zeta potential of PMN suspensions in the presence of polyelectrolytes. In Proceedings of the 7th Ceramic Congress, 2008.

19. Şakar-Deliormanlı, A., Çelik, E. and Polat, M., Adsorption of anionic polyelectrolyte and comb polymers onto lead magnesium niobate. Colloids Surf. A: Physicochem. Eng. Aspects, 2008, 316, 202-209.

20. Brown, G. L. and Garrett, B. S., Latex thickening: interactions between aqueous polymeric dispersions and solutions. J. Appl. Polym. Sci., 1959, 1, 283-295.

21. Nahass, P., Pober, R. L., Rhine, W. E., Robbins, W. L. and Bowen, H. K., Prediction and explanation of aging shrinkage in tape cast ceramic green sheets. J. Am. Ceram. Soc., 1992, 75, 2372-2378.

22. Zupancic, A., Lpasin, R. and Kristoffersson, A., Influence of particle concentration on rheological properties of aqueous $\alpha$-alumina suspensions. $J$. Eur. Ceram. Soc., 1998, 18, 467-477.

23. Rueb, C. J. and Zukoski, C. F., Viscoelastic properties of colloidal gels. $J$. Rheol., 1997, 41, 197-218.

24. Larson, G. R., The Structure and Rheology of Complex Fluids. Oxford University Press, 1999.

25. Bitterlich, B., Lutz, C. and Roosen, A., Rheological characterization of water-based slurries for the tape casting process. Ceram. Int., 2002, 28, $675-683$.
26. Hunter, R. J., Foundations of Colloid Science. Oxford University Press, UK, 1995.

27. Riedl, Z., Szklenarik, G., Zelko, R., Marton, S. and Racz, I., The effect of temperature and polymer concentration on dynamic surface tension and wetting ability of hydroxypropylmethylcellulose solutions. Drug Dev. Ind. Pharm., 2000, 26, 1321-1323.

28. Lewis, J. A., Colloidal processing of ceramics. J. Am. Ceram. Soc., 2000, 83, 2341-2359.

29. Scherer, G. W., Theory of drying. J. Am. Ceram. Soc., 1990, 73, 3-14.

30. Chiu, R. C., Garino, T. J. and Cima, M. J., Drying of granular ceramic films. I. Effect of processing variables on cracking behavior. J. Am. Ceram. Soc., 1993, 76, 2257-2264.

31. Kiennemann, J., Chartier, T., Pagnoux, C., Baumard, J. F., Huger, M. and Lamerant, J. M., Drying mechanism and stress development in aqueous alumina tape casting. J. Eur. Ceram. Soc., 2005, 25, 1551-1564.

32. Rahaman, M. N., Ceramic Processing and Sintering. Marcel Dekker Inc., USA, 1995.

33. Gentil, S., Damjanoviç, D. and Setter, N., $\mathrm{Pb}\left(\mathrm{Mg}_{1 / 3} \mathrm{Nb}_{2 / 3}\right) \mathrm{O}_{3}$ and $(1-x) \mathrm{Pb}\left(\mathrm{Mg}_{1 / 3} \mathrm{Nb}_{2 / 3}\right) \mathrm{O}_{3}-x \mathrm{PbTiO}_{3}$ relaxor ferroelectric thick films: processing and electrical characterization. J. Electroceram., 2004, 12, $151-161$ 